\title{
Extraordinary Absorption of Decorated Undoped Graphene
}

\author{
T. Stauber and G. Gómez-Santos \\ Departamento de Física de la Materia Condensada, Instituto Nicolás Cabrera and Condensed Matter Physics Center (IFIMAC), \\ Universidad Autónoma de Madrid, E-28049 Madrid, Spain \\ F. Javier García de Abajo \\ ICFO-Institut de Ciencies Fotoniques, Mediterranean Technology Park, 08860 Castelldefels (Barcelona), Spain and ICREA-Institució \\ Catalana de Recerca i Estudis Avançats, Passeig Lluís Companys, 23, 08010 Barcelona, Spain
}

(Received 23 October 2013; published 20 February 2014)

\begin{abstract}
We theoretically study absorption by an undoped graphene layer decorated with arrays of small particles. We discuss periodic and random arrays within a common formalism, which predicts a maximum absorption of $50 \%$ for suspended graphene in both cases. The limits of weak and strong scatterers are investigated, and an unusual dependence on particle-graphene separation is found and explained in terms of the effective number of contributing evanescent diffraction orders of the array. Our results can be important to boost absorption by single-layer graphene due to its simple setup with potential applications to light harvesting and photodetection based on energy (Förster) rather than charge transfer.
\end{abstract}

DOI: 10.1103/PhysRevLett.112.077401

Introduction.-The optical properties of graphene have recently been the focus of special attention due to its potential application to nanophotonics and optoelectronics, mainly due to the strong electrical tunability displayed by this material over a broad spectral range down to the infrared [1]. The absorption of a single layer of undoped graphene takes an approximately constant value $\pi \alpha \approx 2.3 \%$ over a wide spectral range and is solely governed by the fine-structure constant $\alpha$ and not by material constants $[2,3]$. For infrared frequencies, this result is readily obtained within the linear Dirac model from the universal conductivity $\sigma=e^{2} / 4 \hbar$, but it also holds for visible light in spite of trigonal warping effects due to a partial cancellation of the enhanced density of states versus the suppressed dipole moment [4]. Since the intrinsic light-matter coupling is given by $\alpha$, one would also expect the absorption of $2 \mathrm{D}$ patterned or decorated graphene to be proportional to this constant.

Despite the low absorption of a single carbon layer, light harvesting based on graphene has been investigated ever since, resulting in different methods to increase the level of absorption based on periodic nanopatterning [5-9], retardation effects [10,11], or placing the graphene in a resonant cavity [12-14]. Additionally, strong absorption can also be driven by auxiliary photoactive materials, such as colloidal quantum dots [15] or semiconducting two-dimensional crystals that transfer electrons or holes into the graphene (e.g., transition metal dichalcogenides such as $\mathrm{MoS}_{2}$ or $\left.\mathrm{WS}_{2}[16]\right)$.

The above proposals are limited because they generally require advanced experimental facilities and operating over a narrow spectral range. In this Letter, we propose a simple method applicable over a wide spectral range using
PACS numbers: 78.67.Wj, 42.25.Bs, 78.20.Ci, 78.70.En

graphene as a photoactive material. The structures under consideration (e.g., randomly depositing nonabsorbing scatterers on top of an undoped graphene layer) only involve relatively modest fabrication techniques, and they can be tested using conventional optical characterization setups. The absorption is mediated by energy transfer into the graphene (Förster effect [17]), which exploits the excellent quenching properties of this material [18-20] to strongly redirect the evanescent field produced by the small scatterers into the absorbing carbon layer. Also crucial for the efficiency of the proposed mechanism is the algebraic dependence of absorption on distance $z$ as $\sim z^{-4}$ for undoped graphene [21-23], in contrast to the exponential decay with the distance of plasmon-driven absorption near doped graphene [24]. This allows us to consider distances $z$ for optimum absorption of the order of (weak scatterers) or well beyond (resonant scatterers) the Fermi wavelength $\lambda_{g}=\lambda v_{F} / c$, where $v_{F} \approx 10^{6} \mathrm{~m} / \mathrm{s}$ is the Fermi velocity and $\lambda$ is the optical wavelength. Alternatively, the increase in absorption associated with the presence of surface scatterers can be qualitatively interpreted as the result of light spending more time near the graphene layer [25].

Absorption driven by an individual dipole.-We first discuss a single nonabsorbing particle and show that in the presence of graphene, the extinction cross section is partially converted into an absorption cross section. The particle is described through a point dipole excited by an incoming light field. In the absence of graphene, the particle scatters light with an extinction cross section $\sigma_{\mathrm{ext}}^{0}=\left(k / \varepsilon_{0}\right) \operatorname{Im}\left\{\alpha_{p}\right\}$, where $k=2 \pi / \lambda$ and $\alpha_{p}$ is the particle polarizability. For a weak scatterer, we can approximate $\alpha_{p}^{-1}=\alpha_{E}^{-1}-i k^{3} / 6 \pi \epsilon_{0}$, where $\alpha_{E}$ is the real 
electrostatic polarizability and the $k^{3}$ term is introduced to satisfy the optical theorem to first order [26], so that we have $\sigma_{\text {ext }}^{0}=\left(\alpha_{E}^{2} / 6 \pi \epsilon_{0}^{2}\right) k^{4}$. In the strong-scatterer limit (e.g., a perfect two level atom), the dipole strength is only limited by radiation reaction and we can approximate $\alpha_{p}^{-1} \approx$ $-i k^{3} / 6 \pi \epsilon_{0}$. This yields $\sigma_{\mathrm{ext}}^{0}=6 \pi / k^{2} \sim \lambda^{2}$, which is large compared with the cross section typically exhibited by metallic nanoparticles.

In the presence of graphene, the decay rate is largely modified and part of the released energy is absorbed by the carbon layer, mainly through nonradiative coupling. The absorbed power is given by $\hbar \omega \gamma_{\mathrm{NR}}$, where $\gamma_{\mathrm{NR}}$ is the nonradiative decay rate. In units of the natural radiative decay rate $\gamma_{0}$, and only considering the longitudinal response, which is dominant at short distances $z \ll \lambda$, we find a characteristic distance dependence [21-23]

$$
\tilde{\gamma}_{\mathrm{NR}}=\frac{\gamma_{\mathrm{NR}}}{\gamma_{0}}=\frac{9 \alpha}{256 \pi^{3}} \frac{1}{(1+\epsilon)^{2}} \frac{1}{(z / \lambda)^{4}},
$$

where $\epsilon$ is the dielectric constant of the substrate, assumed to be real. This nonradiative decay saturates for $z \lesssim \lambda_{g}$, reaching extremely large values $\tilde{\gamma}_{\mathrm{NR}}>10^{6}$.

If the dipole excitation was decoupled from the graphene, the absorption cross section could be largely enhanced by simply bringing the dipole closer to the carbon layer, leading to $\sigma_{\mathrm{abs}} \rightarrow \sigma_{\mathrm{ext}}^{0} \tilde{\gamma}_{\mathrm{NR}}$. However, the oscillating dipole amplitude is affected by the graphene through strong backreaction mediated by evanescent waves. The self-consistent electric field induced at the position of the dipole particle becomes $\mathbf{E}^{\text {ind }}=G \mathbf{p}$, where $G$ is a particle-independent Green function (see the Supplemental Material [27]). This allows us to write the long-wavelength limit of the normal-incidence absorption cross section as

$$
\sigma_{\mathrm{abs}} \approx \frac{k}{\epsilon_{0}}\left(\frac{2}{1+\sqrt{\epsilon}}\right)^{2} \frac{\operatorname{Im}\{G\}}{\left|\alpha_{p}^{-1}-G\right|^{2}},
$$

which is linear in $\operatorname{Im}\{G\} \propto \operatorname{Re}\{\sigma\}$ (i.e., the real part of the graphene conductivity $\sigma$ ). For a weak perfect-conductor disk scatterer (Fig. 1, left panel), we observe a large absorption exceeding $10^{4}$ times the bare disk extinction cross section. The simple expression $\sigma_{\mathrm{abs}} \rightarrow \sigma_{\mathrm{ext}}^{0} \tilde{\gamma}_{\mathrm{NR}}$ works well at large distances, as backreaction effects can be disregarded for weak scatterers. The expected saturation appears at rather small distances, where the dipole model is anyway a poor approximation. For a strong scatterer (Fig. 1, right panel), assuming a perfect dipolar resonance with $\alpha_{p}^{-1}=-i k^{3} / 6 \pi \epsilon_{0}$, we obtain a sizable absorption reaching $25 \%$ of the bare-scatterer extinction cross section. A strongly nonmonotonic behavior with separation is then observed due to backreaction, leading to a maximum absorption at much larger distances than in the weakscatterer regime.

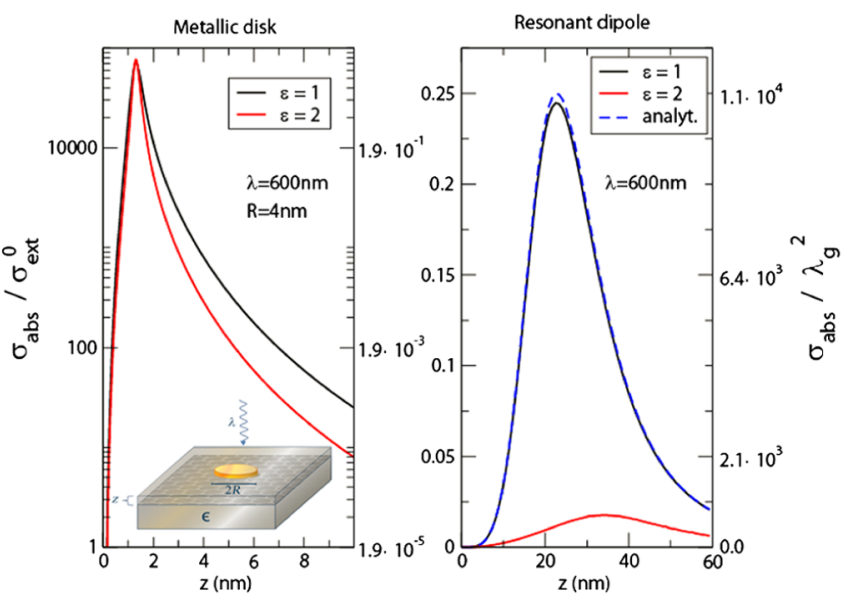

FIG. 1 (color online). Left panel: Dependence of the absorption cross section by a nonabsorbing metallic disk (radius $R=4 \mathrm{~nm}$ ) on its distance to an undoped graphene layer, either suspended $(\epsilon=1)$ or supported on glass $(\epsilon=2)$ for normal incidence. The light wavelength is $\lambda=600 \mathrm{~nm}$. The absorption cross section is given relative to both the bare scatterer extinction (left vertical scale) and the graphene units (right scale) with $\lambda_{g}=\lambda v_{F} / c$. Right panel: Same as the left panel for a resonant dipole. The full theory (solid curves) is compared with the analytical approximation of Eq. (3) (dashed curves).

As anticipated above, the absorption is given by expressions involving the fine-structure constant for distances $z \gtrsim \lambda_{g}=\lambda v_{F} / c$, the regime where the graphene conductivity can be substituted to an excellent approximation by its local, universal limit $\sigma=e^{2} / 4 \hbar$. For example, for a resonant scatterer in front of freestanding graphene, we find (see the Supplemental Material [27])

$$
\frac{\sigma_{\mathrm{abs}}}{\sigma_{\mathrm{ext}}^{0}} \approx \frac{9 \alpha}{1024 \pi^{3}} \frac{(z / \lambda)^{4}}{\left[(z / \lambda)^{4}+\frac{9 \alpha}{1024 \pi^{3}}\right)^{2}},
$$

which is in excellent agreement with our numerical results, as illustrated in Fig. 1 (right panel).

Uniformly decorated graphene.-We consider a layer of uniformly arranged particles that appears to be homogeneous at length scales comparable with the wavelength $\lambda$. In particular, we discuss below both periodic square arrays and completely random distributions, with particle separations $\ll \lambda$. The layer is placed close to a uniform, undoped graphene sheet and illuminated as indicated in Fig. 2(a). The parallel wave vector $\mathbf{k}_{\|}$is fixed by the angle of incidence, leading to a particle dipole amplitude

$$
\mathbf{p}_{n}=\mathbf{p}\left(\mathbf{k}_{\|}\right) e^{i \mathbf{k}_{\|} \cdot \mathbf{r}_{n}},
$$

where $\mathbf{r}_{n}$ is the position of particle $n$. Then, we can write

$$
\mathbf{p}\left(\mathbf{k}_{\|}\right)=\left[\boldsymbol{\alpha}_{p}^{-1}-\mathfrak{G}\left(\mathbf{k}_{\|}\right)\right]^{-1} \mathbf{E}^{\mathrm{ext}},
$$

where $\mathbf{E}^{\text {ext }}$ is the external electric field, 
(a)

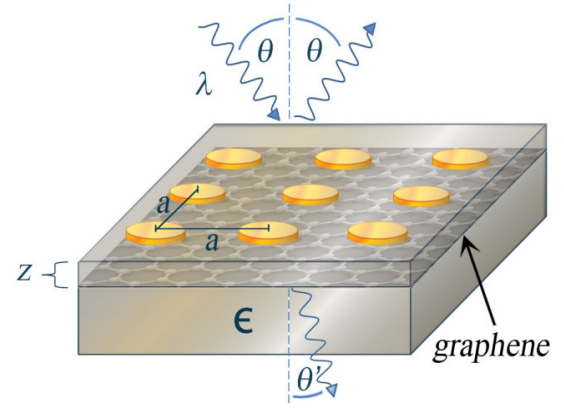

(b)

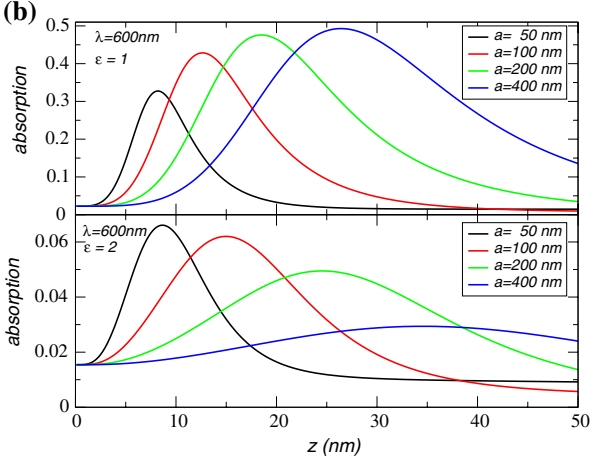

(c)

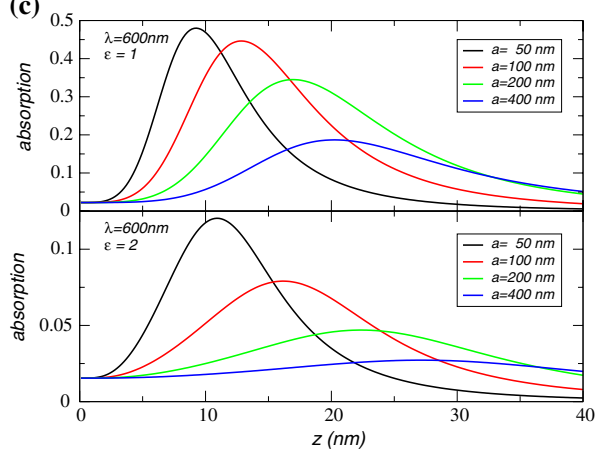

FIG. 2 (color online). (a) We consider the reflection and transmission of light (wavelength $\lambda$, incident angle $\theta$ ) in a planar structure consisting of a square array of electric-dipole particles (e.g., metallic disks of radius $R$ ) placed at a distance $z$ from an undoped graphene layer, which is in turn on top of a substrate of dielectric constant $\epsilon$. (b) Distance dependence of absorption of light at normal incidence for a square array of resonant particles and different lattice periods $a$, with $\lambda=600 \mathrm{~nm}$ and either supported ( $\epsilon=2$, bottom) or self-standing ( $\epsilon=1$, top) graphene. (c) Same as (b) for randomly distributed particles with several densities $\rho=a^{-2}$.

$\mathfrak{G S}\left(\mathbf{k}_{\|}\right)=\int \frac{d^{2} \mathbf{Q}}{(2 \pi)^{2}}\left[\frac{S\left(\mathbf{Q}-\mathbf{k}_{\|}\right)}{\rho}\left[1+\mathfrak{r}(\mathbf{Q}) e^{2 i k_{z} z}\right]-1\right] \mathfrak{g}(\mathbf{Q})$

describes the dipole-dipole interaction, $k_{z}=\sqrt{k^{2}-Q^{2}}, \rho$ is the particle density, $\mathfrak{r}$ is the diagonal graphene (plus substrate) reflection matrix depending on the polarization of the incident wave, and $\mathfrak{g}$ is the vacuum Green function. The explicit definitions are given in the Supplemental Material [27].

All information on particle positions is contained in $\mathcal{S}$, the Fourier transform of the dipole-pair correlation function (see below). The absorbance can then be calculated from the power absorbed per unit area by graphene (see the Supplemental Material [27]).

Periodic particle array.-The structure factor in a periodic array reduces to the contribution from reciprocal lattice vectors $\mathbf{G}$

$$
S(\mathbf{Q})=(2 \pi \rho)^{2} \sum_{\mathbf{G}} \delta(\mathbf{Q}-\mathbf{G})
$$

The integral in Eq. (6) is then transformed into a sum over discrete vectors $\mathbf{Q}=\mathbf{k}_{\|}+\mathbf{G}$, which we evaluate for square arrays of period $a$ (see Refs. [27,28] for more details).

In the nondiffractive regime (i.e., for $\lambda>a$ ), the absorbance reduces to

$$
\mathcal{A}_{j}=1-\left|\tilde{r}_{j}\right|^{2}-\left|\tilde{t}_{j}\right|^{2} \frac{k_{z}^{\prime}}{k_{z}}
$$

where $j=p, s$ indicates the light polarization. The corresponding Fresnel coefficients $r_{j}$ and $t_{j}$ are the sum of direct (without particle dipoles) and radiated (superindex d) contributions $\tilde{r}_{j}=r_{j}^{d}+r_{j}$ and $\tilde{t}_{j}=t_{j}^{d}+t_{j}$, which we derive from Eq. (6) following the methods of Ref. [7].
Explicit expressions for these coefficients are given in the Supplemental Material [27].

For weak scatterers, the absorption is small and quickly vanishing with the distance between the array and the graphene (see the Supplemental Material [27]). We thus concentrate on the more interesting case of resonant dipoles. Results for several lattice densities close to either suspended or silica-supported graphene are shown in Fig. 2(b). One observes a large enhancement in absorption, nearing $\sim 50 \%$ for freestanding graphene. Again, the nonmonotonic behavior with distance originates in the screening effect of graphene reflection at small separations. Particularly striking is the behavior with particle density $\rho$ for freestanding graphene [Fig. 2(b), top]: the overall absorption increases with decreasing density over a significant range of distances where absorption is high. As the absorption coefficient $\mathcal{A}$ is dominated by evanescent modes, the following expression can be obtained in analogy with Eq. (2) under normal incidence:

$$
\mathcal{A} \sim \mathcal{A}_{\mathrm{ev}} \approx \rho \frac{k}{\epsilon_{0}}\left(\frac{2}{1+\sqrt{\epsilon}}\right)^{2} \frac{\operatorname{Im}\left\{\Delta G_{\mathrm{ev}}\right\}}{\left|\alpha_{p}^{-1}-G\right|^{2}}
$$

where $\Delta G_{\mathrm{ev}}$ is the evanescent-wave contribution to Eq. (6) with $|\mathbf{Q}|>k$. From Eq. (9), one would naively expect a linear monotonic dependence with density at fixed distance. Notice, however, that $\Delta G_{\mathrm{ev}}$ also depends on density. In fact, Eq. (7) amounts to a discrete sampling of the integral in Eq. (6), from which the specular contribution is removed. The exponential term $e^{i 2 k_{z} z}\left(=e^{-2\left|k_{z}\right| z} \approx e^{-2|\mathbf{G}| z}\right.$ for $\mathbf{G} \neq 0$ ) in Eq. (6) effectively limits the sum to $|\mathbf{G}| \lesssim 1 / z$, whereas the lowest (evanescent) contributing term is $\left|\mathbf{G}_{1}\right|=2 \pi / a$. Therefore, a reduction in particle density (and hence, also in $\left|\mathbf{G}_{1}\right|$ ) produces an increase in the number of evanescent modes effectively contributing to absorption. 
Disordered layer of dipoles.-In a totally disordered array, the structure factor becomes

$$
S(\mathbf{Q})=(2 \pi \rho)^{2} \delta(\mathbf{Q})+\rho .
$$

The dipolar response now reduces to the diagonal elements

$$
\left(\alpha_{p}^{-1}-G\right)_{\|, \perp}=\alpha_{p}^{-1}-G_{\|, \perp}^{0}-\Delta G_{\|, \perp},
$$

where $G^{0}$ is the contribution from the $\mathbf{G}=0$ specular term and $\Delta G$ originates in the continuum of evanescent modes, as given by

$$
\Delta G_{\|}=\Delta G_{\perp} / 2=\frac{-1}{(2 \pi)^{2}} \int d^{2} \mathbf{Q} r_{p}^{\mathrm{nf}}(\mathbf{Q}) \mathfrak{g}_{x x}(\mathbf{Q}) e^{-2 Q z} .
$$

Here, we use a nonretarded approximation for the reflection coefficient $r_{p}^{\mathrm{nf}}(\mathbf{Q})$, consistent with the short distances $z \ll \lambda$ considered.

As expected, the absorption for weak scatterers (not shown) is very similar in ordered and disordered lattices. For the resonant case, the absorption is shown in Fig. 2(c). Although broadly similar, several differences are noticeable between ordered and random arrays, especially in the low and high particle-density limits. This comparison is highlighted in Fig. 3 for freestanding graphene, showing that random arrays produce more absorption than ordered ones at high densities, reaching almost 50\%. The opposite happens at low densities, where ordered arrays produce higher absorption, again around 50\%.
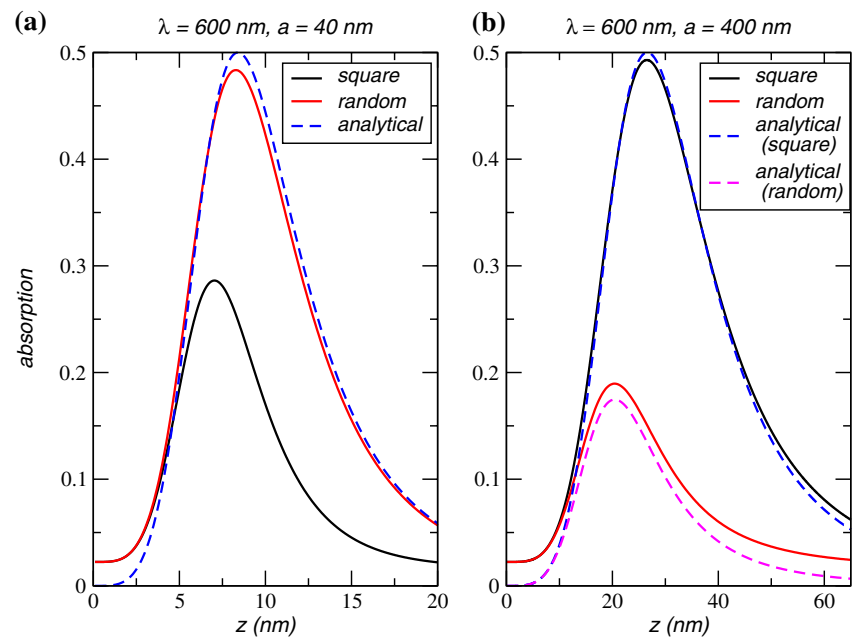

FIG. 3 (color online). Left panel: Dependence of absorption on the distance $z$ between either an ordered or a disordered particle array with respect to the graphene [see Fig. 2(a)]. The particle density is $\rho=40^{-2} \mathrm{~nm}^{-2}$ in both cases, the graphene is selfstanding, and the light wavelength is $\lambda=600 \mathrm{~nm}$. Full results are compared with the analytical expression of Eq. (13). Right panel: Same as the left panel for a density $\rho=400^{-2} \mathrm{~nm}^{-2}$.
We compare the absorbance at resonance frequency of both ordered and disordered freestanding graphene with the analytical approximation

$$
\mathcal{A} \approx \frac{2 B(z / \lambda)^{4}}{\left[C(z / \lambda)^{4}+B\right]^{2}}
$$

with $B=3 \alpha /\left(256 \pi^{2} \rho \lambda^{2}\right)$ and either $C=1$ (ordered array) or $C=1+4 \pi /\left(3 \rho \lambda^{2}\right)$ (disordered array). Equation (13) is obtained from Eq. (11) by setting $\operatorname{Re}\left\{\alpha_{p}^{-1}\right\}=0$ (resonant case), using the local universal value for the graphene conductivity $\sigma=e^{2} / 4 \hbar$, valid for distances $z \gtrsim \lambda_{g}=$ $\lambda v_{F} / c$. Interestingly, only $\alpha$, the scaled distance $z / \lambda$, and the number of particles per square wavelength $\rho \lambda^{2}$ appear in Eq. (13); i.e., no material constants of graphene enter and the proposed mechanism is independent of the dipole resonance $\Omega=2 \pi c / \lambda$ due to graphene's broadband properties. The linewidth of the isolated dipole resonance $\gamma \ll \Omega$ can further be strongly enhanced due to absorption in the graphene layer to $\gamma_{\mathrm{abs}}=(3 / 2 \pi) \rho \lambda \gamma$, yielding extremely large values for high concentrations and long resonance wavelengths (see the Supplemental Material [27]).

Figure 3 shows the analytical result to be an excellent approximation for random distributions at high densities, whereas the numerically calculated absorption of ordered arrays is substantially lower due to the detrimental role of the discrete wave-vector distribution of evanescent modes in this limit. In contrast, it is the higher absorption of ordered arrays that almost perfectly matches the corresponding analytical result at low densities. This can be understood as follows: the actual particle resonance condition $\operatorname{Re}\left\{\alpha_{p}^{-1}\right\}=0$ implies $\alpha_{p}^{-1}=-i k^{3} / 6 \pi \epsilon_{0}$ in Eq. (11) for the disordered array, whereas such an imaginary contribution is absent for the ordered array due to the layer dynamical self-screening of radiation reaction [28]. In more physical terms, diffuse scattering into radiative modes, absent in (nondiffractive) ordered arrays, persists in disordered arrays with relative importance increasing at low densities, thus producing a stronger radiative response without significantly contributing to absorption.

We remark that the maximum absorption attainable according to Eq. (13) is 50\%, a value closely approached by the numerical results in the regimes discussed above. This is the absolute absorption limit of a thin layer in vacuum [28], which applies to our structures because $z \ll \lambda$. The dipole arrangements considered, periodic and totally disordered, represent opposite extremes. Therefore, we expect similar absorption enhancements for intermediate situations, such as partially periodic structures, with some degree of disorder, and also for quasicrystalline arrangements.

Summary.-We have shown that the absorption of a single, undoped graphene layer can be dramatically enhanced (up to $\sim 50 \%$ in the self-standing configuration) by decorating it with nonabsorbing small particles. Both 
ordered and disordered particle arrays can produce such an effect. In a plausible experimental realization, one can deposit small dielectric particles on a graphene layer. Such high absorption is possible over a wide spectral range from the visible to the infrared. A counterintuitive increase in absorption with decreasing particle density is predicted for ordered arrays, particularly when resonant particles are considered, which we understand in terms of the effective number of contributing evanescently diffracted orders. An analytical expression in terms of fundamental units has been derived for resonant dipoles, exhibiting a maximum of $50 \%$, which is the intrinsic limit for the absorption of a thin layer. To the best of our knowledge, this is the first system to display such behavior for normal incidence without explicitly relying on plasmonic effects [29].

Our analysis can be straightforwardly applied to other quasi-2D materials by simply using an appropriate expression for the conductivity $\sigma$, i.e., by replacing $\alpha$ by $\sigma / \pi \epsilon_{0} c$ throughout our expressions [30]. The universality of the broadband constancy of the conductivity outside gap regions [31] then yields equivalently universal absorption results in decorated planar absorbing layers. Semiconductor heterostructures and multilayer graphene can thus be directly analyzed in this way. Finally, our work provides an alternative strategy to induce photocurrents mediated by energy rather than charge transfer in graphene-based heterostructures, relying on the large optical quenching produced by undoped graphene on nearby optical emitters.

This work has been supported in part by FCT (PTDC/ FIS/101434/2008, PTDC/FIS/113199/2009), MIC (FIS2010-21883-C02-02, FIS2012-37549-C05-03), and the EC (Graphene Flagship CNECT-ICT-604391 and FP7-ICT-2013-613024-GRASP).

[1] F. Bonaccorso, Z. Sun, T. Hasan, and A. C. Ferrari, Nat. Photonics 4, 611 (2010).

[2] R. R. Nair, P. Blake, A. N. Grigorenko, K. S. Novoselov, T. J. Booth, T. Stauber, N. M. R. Peres, and A. K. Geim, Science 320, 1308 (2008).

[3] K. F. Mak, M. Y. Sfeir, Y. Wu, C. H. Lui, J. A. Misewich, and T. F. Heinz, Phys. Rev. Lett. 101, 196405 (2008).

[4] T. Stauber, N. M. R. Peres, and A. K. Geim, Phys. Rev. B 78, 085432 (2008).

[5] L. Ju, B. Geng, J. Horng, C. Girit, M. Martin, Z. Hao, H. A. Bechtel, X. Liang, A. Zettl, Y. R. Shen, and F. Wang, Nat. Nanotechnol. 6, 630 (2011).

[6] T. Echtermeyer, L. Britnell, P. Jasnos, A. Lombardo, R. Gorbachev, A. Grigorenko, A. Geim, A. Ferrari, and K. Novoselov, Nat. Commun. 2, 458 (2011).

[7] S. Thongrattanasiri, F. H. L. Koppens, and F. J. García de Abajo, Phys. Rev. Lett. 108, 047401 (2012).

[8] A. Y. Nikitin, F. Guinea, and L. Martin-Moreno, Appl. Phys. Lett. 101, 151119 (2012).
[9] N. M. R. Peres, Y. V. Bludov, A. Ferreira, and M. I. Vasilevskiy, J. Phys. Condens. Matter 25, 125303 (2013).

[10] Y. V. Bludov, M. I. Vasilevskiy, and N. M. R. Peres, Europhys. Lett. 92, 68001 (2010).

[11] G. Gómez-Santos and T. Stauber, Europhys. Lett. 99, 27006 (2012).

[12] A. Ferreira, N. M. R. Peres, R. M. Ribeiro, and T. Stauber, Phys. Rev. B 85, 115438 (2012).

[13] M. Furchi, A. Urich, A. Pospischil, G. Lilley, K. Unterrainer, H. Detz, P. Klang, A. M. Andrews, W. Schrenk, G. Strasser, and T. Mueller, Nano Lett. 12, 2773 (2012).

[14] G. Pirruccio, L. Martín Moreno, G. Lozano, and J. Gómez Rivas, ACS Nano 7, 4810 (2013).

[15] G. Konstantatos, M. Badioli, L. Gaudreau, J. Osmond, M. Bernechea, F. P. G. de Arquer, F. Gatti, and F. H. L. Koppens, Nat. Nanotechnol. 7, 363 (2012).

[16] L. Britnell, R. M. Ribeiro, A. Eckmann, R. Jalil, B. D. Belle, A. Mishchenko, Y.-J. Kim, R. V. Gorbachev, T. Georgiou, S. V. Morozov, A. N. Grigorenko, A. K. Geim, C. Casiraghi, A. H. C. Neto, and K. S. Novoselov, Science 340, 1311 (2013).

[17] T. Förster, Ann. Phys. (Berlin) 437, 55 (1948).

[18] J. Kim, L. J. Cote, F. Kim, and J. Huang, J. Am. Chem. Soc. 132, 260 (2010).

[19] Z. Chen, S. Berciaud, C. Nuckolls, T. F. Heinz, and L. E. Brus, ACS Nano 4, 2964 (2010).

[20] K. P. Loh, Q. Bao, G. Eda, and M. Chhowalla, Nat. Chem. 2, 1015 (2010).

[21] R. S. Swathi, and K. L. Sebastian, J. Chem. Phys. 130, 086101 (2009).

[22] G. Gómez-Santos and T. Stauber, Phys. Rev. B 84, 165438 (2011).

[23] L. Gaudreau, K. J. Tielrooij, G. E. D. K. Prawiroatmodjo, J. Osmond, F. J. García de Abajo, and F. H. L. Koppens, Nano Lett. 13, 2030 (2013).

[24] K. A. Velizhanin and A. Efimov, Phys. Rev. B 84, 085401 (2011).

[25] H. A. Atwater and A. Polman, Nat. Mater. 9, 865 (2010).

[26] B. T. Draine and P. J. Flatau, J. Opt. Soc. Am. A 11, 1491 (1994).

[27] See Supplemental Material at http://link.aps.org/ supplemental/10.1103/PhysRevLett.112.077401 where we present detailed derivations of the analytical results presented in this paper, as well as discussions of resonant detuning and linewidth, and the application to fluorescent molecules.

[28] F. J. García de Abajo, Rev. Mod. Phys. 79, 1267 (2007).

[29] For grazing incidence, absorption of $50 \%$ without the dipole layer is possible for $s$-polarized light; see, in the case of graphene, A. Gutiérrez-Rubio, T. Stauber, and F. Guinea, J. Opt. 15, 114005 (2013).

[30] For (few-) $n$-layer graphene, $\alpha$ is replaced by $n \alpha$, valid in the optical regime.

[31] H. Fang, H. A. Bechtel, E. Plis, M. C. Martin, S. Krishna, E. Yablonovitch, and A. Javey, Proc. Natl. Acad. Sci. U.S.A. 110, 11688 (2013). 